\title{
Regulación internacional de Internet: una aproximación desde las capas de la red
}

\author{
International Internet Regulation: \\ A Technical Approach from Network Layers
}

\author{
FRANCISCO VERA HOTT \\ Abogado, Privacidad y Seguridad SpA
}

RESUMEN El estudio de la regulación internacional de Internet requiere de la sistematización de sus órganos y regulaciones desde una perspectiva que exceda el análisis jurídico. El presente artículo presenta un análisis de estas regulaciones internacionales a partir del concepto de capas de la red, buscando relaciones entre la estructura técnica de Internet, compuesta por capas de infraestructura, lógica y de contenidos, y su ordenamiento institucional y normativo, bajo el entendido que las dimensiones técnica y jurídica se encuentran íntimamente relacionadas entre sí, revisando instituciones y normas clave en cada capa de la red. Además, se explora la teoría de separación de las capas como un marco capaz de ofrecer principios básicos para la regulación internacional de Internet.

PALABRAS CLAVE Gobernanza de Internet, regulación internacional de Internet, teoría de las capas, capas de la red, regulación internacional de telecomunicaciones, UIT, ICANN, CMSI, FGI.

ABSTRACT The study of international Internet regulation requires of the systematization of its regulations and organs from a perspective that exceeds the legal analysis. This article presents an analysis of these interna- 
tional regulations starting from the network layers framework, looking for relations between the technical structure of the Internet, composed by an infrastructure layer, a logical layer and a content layer; and its institutional and regulatory arrangement, all the above under the assumption that the technical and legal dimensions are closely interrelated, and checking for key institutions and regulations in each network layer. Besides, a layers separation theory is explored, as a framework capable to offer principles for a basic international Internet regulation.

KEYWORDS Internet governance, international Internet regulation, layers theory, network layers, international telecommunications regulation, ITU, ICANN, WSIS, IGF.

\section{INTRODUCCIÓN}

Una de las falacias más recurrentes respecto a la regulación de Internet es que ésta carecería de una suficiente regulación, con lo que las realidades que se configuran a partir de la red se encontrarían prácticamente en un estado de naturaleza, sin que existan normas que rijan a los usuarios de la red. Esta regulación, sin embargo, existe y tiene plena vigencia, tanto porque las normas jurídicas destinadas a gobernar lo que podríamos denominar «mundo real» también reciben aplicación en Internet, o bien porque estas realidades que se configuran en la red, aun cuando no provengan de fuentes jurídicas formales, sí están reguladas por normas de diversa índole.

Bajo la premisa anterior, Internet sí estaría regida por regulaciones de variada naturaleza. Estas reglas entrañan un altísimo nivel de complejidad por su carácter descentralizado en lo técnico y lo legal, comprendiendo una serie de diversas tecnologías y jurisdicciones nacionales que configuran un marco normativo a Internet.

Debido a que Internet es una tecnología de alcance global, reviste un particular interés el análisis de cómo se regula a nivel internacional. No obstante, un análisis de esta naturaleza, como ya adelantamos, no puede enmarcarse solamente en lo jurídico, sino que debe combinar una revisión de la estructura técnica de la red y de los arreglos normativos técnicos, legales y políticos que tienen lugar en su interior. 
Estos arreglos normativos pueden también resumirse en el concepto de gobernanza de Internet, concepto que abarca los múltiples actores, instituciones, foros, normas y prácticas que controlan y gobiernan la red. Proveer una definición formal y universalmente aceptada de este término resulta hoy en día una tarea sumamente difícil y, hasta cierto punto, infructuosa, atendido que cada una de las definiciones que existen responde a los intereses y enfoque de un autor específico. No obstante, y para evitar una excesiva vaguedad en el concepto, tendremos en mente los cuatro parámetros propuestos en el libro The Global War for Internet Governance (DeNardis, 20I4: I8) para delimitar el concepto, y que son:

I. el estudio de la gobernanza de Internet es distinto al estudio de uso de Internet;

2. los temas de gobernanza de Internet se relacionan con la arquitectura única de Internet, más allá del diseño y aplicación de regulaciones en general para las tecnologías de la información;

3. la gobernanza de Internet se extiende más allá de instituciones como ICANN (Internet Corporation for Assigned Names and Numbers) e incluye regulaciones privadas de la industria, nacionales, tratados internacionales y el diseño de la arquitectura técnica; y

4. la gobernanza de Internet incluye formas de control que apuntan a la interoperabilidad y el acceso al conocimiento, pero al mismo tiempo aquellas técnicas orientadas a reducir la libertad de Internet.

Una vez definido el ámbito geográfico (global) y normativo (gobernanza de Internet) del presente trabajo, queda proponer un marco que permita sistematizar el análisis, pero también darle un sentido y aventurar algunas conclusiones.

Para cumplir ese objetivo, el presente trabajo sistematizará la regulación a nivel internacional de Internet desde una perspectiva técnica, basándose en la clasificación de Internet en capas de infraestructura física, lógica y de contenidos, identificando en cada una de las capas las normas internacionales relevantes para comprender y analizar las mismas, desde su regulación jurídica o la falta de ésta, poniendo énfasis en los temas que se encuentran actualmente en discusión. 
Ciertamente, abarcar plenamente la regulación internacional de Internet, empleando un marco amplio de análisis derivado del concepto de gobernanza de Internet, desde una perspectiva técnica, es una tarea que excede por mucho la profundidad que puede tener un trabajo de estas características. Debido a lo anterior, tras presentar sucintamente los aspectos técnicos de Internet y sus capas y los principios regulatorios que deben seguirse a su respecto, el análisis se centrará en aquellas instituciones y normas que presentan los mayores desafíos para el futuro de la red, ya sea por su inherente importancia o por la actualidad en su debate.

\section{INTERNET Y SU FUNCIONAMIENTO EN CAPAS}

Analizar un determinado fenómeno a través del análisis de sus capas no es una idea nueva. De hecho, la definición más frecuente de la palabra análisis implica la separación de una cosa en diversas unidades.

Internet puede definirse como una red global de redes que se ha convertido en una plataforma para innovaciones radicales respecto a diversas actividades humanas (Lessig, 200I: xxi). La masificación de Internet supone complejos y múltiples desafíos regulatorios a nivel global. Muchos de esos desafíos surgen del funcionamiento de la red, que ha sido conceptualizado como un sistema de múltiples capas que gestionan diferentes funciones en la red.

El antecedente más conocido de un modelo de comunicación estructurado en capas proviene de un modelo conocido como OSI, que conceptualiza y estandariza las funciones de un sistema de comunicaciones separándolo en capas abstractas, a partir de un proyecto desarrollado en la Organización Mundial para la Estandarización (en inglés, ISO), y mantenido bajo la especificación ISO/IEC 7498-I (OSI Model 20I4).

Ahora, ¿cuáles son estas capas y qué hace cada una? El modelo OSI consta de 7 capas:

- Capa física: define las especificaciones físicas y eléctricas de la conexión de datos, incluyendo los medios de transmisión y detalles sobre pines, voltajes, etcétera.

- Capa de enlace de datos: provee un enlace confiable entre dos nodos conectados.

- Capa de red: entrega los medios para la transferencia de secuencias 
de datos entre los nodos conectados a una red, sin gestionar la calidad ni integridad de la información.

- Capa de transporte: se encarga de controlar la transferencia de información entre dos nodos, controlando y manteniendo la calidad de los datos. El mejor ejemplo de esta capa es el protocolo de transmisión y control de datos, conocido como TCP, que normalmente funciona en conjunto con la tecnología de paquetes de datos, dando origen al protocolo TCP/IP, que es la piedra angular para la estructura de red que da origen a Internet, que constituye un sistema de capas simplificado.

- Capa de sesión: controla el diálogo entre los computadores. Establece, gestiona y termina las conexiones entre las aplicaciones locales (del computador propio) y remotas (del otro computador).

- Capa de presentación: permite dar sintaxis a la información, entregando contexto a la misma. Permite transmitir datos estructurados (por ejemplo: una tabla), o cifrados, otorgando un nivel de independencia entre el contenido y su representación.

- Capa de aplicación: para el modelo OSI, esta capa es la más cercana al usuario, permitiéndole interactuar directamente con la aplicación computacional. Un ejemplo es el protocolo de transferencia de hipertexto, que permite al usuario interactuar con la red Internet a través de lo que hoy conocemos como sitios web.

Este modelo no es más que una abstracción de las etapas y protocolos que intervienen en una transmisión de datos en red, y esa misma abstracción es la que distingue la información en formato analógico del digital. Salvo la capa física, de la segunda a la séptima capa son principalmente programas de computación que muchas veces no se distinguen realmente entre sí, salvo para efectos de análisis.

Otra versión del modelo de capas es el modelo TCP/IP, que es una versión más simple del modelo OSI y consiste en una abstracción de capas, diseñada para interconectar varias redes que existían a la fecha de su creación. Estas capas son cinco (Solum y Chung, 2004):

- Capa física: tal como en el modelo OSI, es el medio físico sobre el que la transferencia de datos tiene lugar. 
- Capa de enlace: también como en el modelo OSI, es el que provee un enlace entre los nodos conectados. En la práctica, en Internet es el rol que tienen los protocolos que conectan un nodo a la red, como Ethernet en el caso de una tarjeta de red, o IEEE 802, más conocido como Wi-Fi, que conectan un computador a la red.

- Capa de red: esta capa es la que se denomina propiamente IP, o Internet Protocol, y es la que gestiona el movimiento de paquetes de datos a través de la red, definiendo la forma en que estos paquetes se mueven. El Internet Protocol permite que los paquetes de datos viajen entre puntos diferentes de la red, calculando una ruta óptima viajando entre distintas redes de datos, lo que dota a la red de una gran capacidad de respuesta a fallos.

- Capa de transporte: esta capa es la que procesa los datos y los entrega a la capa de aplicación.

- Capa de aplicación: maneja los detalles de una aplicación particular, por ejemplo HTTP para la web, IMAP, SMTP y POP para el correo electrónico, o FTP para el intercambio de archivos.

En ambos modelos las capas están organizadas jerárquicamente. Cuando la información fluye, pasa desde la capa de contenidos hacia las capas inferiores, donde se convierte en paquetes de datos para luego viajar por la capa física, donde se desplaza horizontalmente hacia su destino, donde los paquetes de datos vuelven a transformarse en contenidos. Bajo este diseño de red es que surge el principio de punto a punto, que se basa en la definición de Internet como una plataforma en donde las capas que se encuentran bajo la aplicación funcionan de manera independiente y neutral a cualquier contenido, y cualquiera puede desarrollar aplicaciones en base al protocolo TCP/IP, con la expectativa que ellas puedan ser ejecutadas en cualquier nodo de la red, sin que las capas inferiores interfieran en ese proceso. Sin embargo, dicho principio no implica que dicha interferencia sea imposible en dichos niveles.

No obstante lo anterior, los modelos analizados están centrados en la transmisión de información dentro de las redes desde una perspectiva técnica y no en la naturaleza de esos contenidos. En ese sentido, para efectos de nuestro análisis posterior, seguiremos a Benkler (2000) y su modelo simplificado en tres capas: 
- Capa física: tal como en los modelos anteriores, comprende el medio físico en que se desenvuelve la conexión de un nodo a la red.

- Capa lógica: en el modelo de Benkler, se agrupan las capas de enlace, red, transporte y aplicación del protocolo TCP/IP.

- Capa de contenidos: en este modelo se reconoce y añade una capa clave para el análisis legal, consistente en los contenidos que circulan por la red, tales como textos, música, fotos, videos, etcétera.

Es en base a este modelo que efectuaremos el análisis de la regulación internacional de Internet. Sin embargo, antes tenemos que referirnos a la relación entre código (capas) y regulación.

\section{DEL CÓDIGO A LA REGULACIÓN}

«Gobiernos del mundo industrializado, gigantes de carne y acero, vengo del ciberespacio, el nuevo hogar de la mente. A nombre del futuro, les pido a ustedes, que vienen del pasado, que nos dejen solos. No son bienvenidos entre nosotros. Ustedes no tienen soberanía en el lugar que

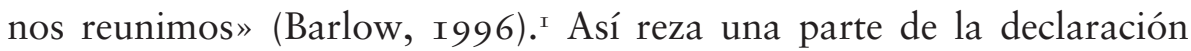
de independencia del ciberespacio, a estas alturas todo un clásico en lo que respecta a manifiestos ideológicos vinculados con las nuevas tecnologías. Escrita por John Perry Barlow el año I996, rescata el concepto de ciberespacio acuñado por William Gibson el año I984 en su obra Neuromante, y lo declara libre de toda injerencia de parte de los poderes tradicionales.

Esta declaración, famosa en su propio mérito, es citada en el libro Code 2.0 (Lessig, 2006) para ilustrar la aproximación ideológica que existió respecto a Internet en sus albores: un lugar que está fuera del control humano. Sin embargo, señala Lessig, el control también existe en este espacio, y ese control viene dado por las fundaciones sobre las que se construye una realidad específica. En este caso, el control viene dado por el «código» con que fue escrito el ciberespacio, y ese «código» es su ley.

Lo anterior tiene dos consecuencias prácticas que han determinado

I. Traducción libre. Texto disponible en <https://projects.eff.org/ barlow/Declaration-Final.html>. 
el panorama internacional de la regulación de Internet: en primer lugar, que el punto de partida viene dado por los desarrollos técnicos que le dieron origen; y, en segundo lugar, que para reescribir esta regulación del ciberespacio no bastaba con negociar y acordar normas jurídicas: estas normas jurídicas debían estar reflejadas a su vez en este "código».

La primera consecuencia práctica (que el punto de partida viene dada por el «código») implica que si se pretende regular Internet sin tener en consideración la estructura técnica (el código) con que se construyó la red, se está ignorando la regulación inicial de ésta, y el resultado de este proceso puede ser imposible de implementar por razones técnicas, o menoscabar los atributos que Internet ofrece, como su apertura, eficiencia, interoperabilidad o resistencia a fallos; donde algunos de ellos obedecen a la teoría de las capas, como veremos.

La segunda consecuencia es también interesante: la única forma de reflejar las regulaciones legales en la red es reflejándolas en el código, convirtiendo a éste en agente de la aplicación de la ley.

Mientras el código es una realidad y es la ley que impera en el ciberespacio, ${ }^{2}$ las normas jurídicas deben comprender ese código y responder al mismo o modificarlo para ser efectivas. En ese sentido, la regulación de Internet deberá considerar estas circunstancias preexistentes, adoptando el desafío de preservar la integridad de la red, mientras persigue los objetivos de política pública propios de su existencia.

En otras palabras, para preservar la red, la regulación deberá conocer el código y hablar en ese idioma. De otra forma, o la regulación se torna irrelevante o se corre el riesgo de romper el diseño de la misma, configurando un sistema diferente - y muy probablemente más ineficienteque lo que hoy conocemos como Internet.

Este principio ha sido desarrollado como el "principio de las capas», que consiste en que los reguladores de Internet no deberían crear o adoptar regulaciones que violen la integridad de las capas de no existir un interés superior a regular, siempre considerando alternativas menos gravosas (Solum y Chung, 2003:43).

Los autores detrás de este principio continúan señalando que, para

2. Sin excluir, por supuesto, fuerzas como las normas sociales y el mercado, entre otros agentes relevantes que configuran el sistema normativo en la red. Sin embargo, el foco de este trabajo es analizar el impacto del código en la regulación internacional de Internet. 
que este principio funcione, se requieren dos principios auxiliares o corolarios:

- El principio de separación de las capas, consistente en que los reguladores de Internet no adopten medidas que requieran que una capa de Internet se base en información disponible en otra capa, manteniendo cada capa en una especie de silo. Este principio va de la mano con el principio de punto a punto y la transparencia de las capas.

- El principio de minimización del cruce entre capas, que establece que si existe un interés superior que requiera normar más de una capa, los reguladores deberán adoptar medidas que minimicen la distancia entre la capa en la que se pretenden radicar los efectos y aquella objeto de la regulación, que se traduce en cruzar la menor cantidad de capas. Este principio es sumamente relevante al momento de analizar modalidades de filtrado de Internet en los extremos (Yoo, 2013: 19).

Los principios analizados tienen una serie de aplicaciones prácticas, más allá de aquellas donde su influencia es evidente, como en el caso del debate relativo a la neutralidad de la Red. Por ejemplo, el análisis por capas y la aplicación de estos principios permite la evolución de la regulación en materia de telecomunicaciones, dando un tratamiento unificado a la capa física, sin importar el medio por el cual se provea Internet, y propiciando soluciones innovadoras para proveer esta conexión (Werbach, 2002: 2; Whitt, 2004: 6I 5).

El principio de separación de capas también se manifiesta en recomendaciones de políticas públicas en la Organización para la Cooperación y el Desarrollo Económicos (OCDE). En la Recomendación del Consejo de la OCDE para el desarrollo de políticas de Internet, uno de los principios se denomina «Promover la naturaleza abierta, distribuida e interconectada de Internet», y profundiza señalando: «Los roles, apertura y competencias de las organizaciones que gobiernan estándares para las diferentes capas de Internet, deben ser reconocidas y su contribución volcarse como elementos técnicos en los objetivos de política pública» (OECD, 20II).

Estos principios pueden también convertirse en una metodología de 
resolución de casos, especialmente cuando entran en juego derechos fundamentales, y se conocen como la «metodología de las capas», que tal como su nombre lo sugiere, consiste en analizar los casos separando sus efectos capa por capa de la red, considerando los efectos de la decisión en cada capa en lugar de referirse a Internet como un todo monolítico, y aprovechándose de las capas para maximizar el alcance de los derechos y minimizar sus restricciones (Bailey, 2004: I7).

$\mathrm{Al}$ analizar qué capa es afectada y su relación con cada derecho fundamental, se evitan decisiones que tratan a Internet como una tecnología monolítica, y por consiguiente, afectar capas de la red que puedan crear consecuencias potencialmente más adversas que la solución propuesta. Por ejemplo, en Argentina durante el año 2010, en relación con un caso de publicación de información reservada, un tribunal ordenó el bloqueo no de contenidos específicos, sino que de todo un servidor de Internet, que resultó ser de un servicio (Blogger) que alojaba miles de otros sitios web, resultando en un grave caso de afectación al derecho de libertad de expresión. ${ }^{3}$

Existen, sin embargo, algunas críticas a este modelo de capas, principalmente porque define ex ante un concepto de capas que puede extenderse y condicionar el análisis económico de un mercado, sin profundizar cuáles son los mercados relevantes o los impactos económicos de ciertas regulaciones, especialmente cuando la capa física se regula en demasía, mediante la preferencia explícita de una solución tecnológica sobre el resto (Reed, 2006: 296). En otras palabras, las críticas a este modelo se generan por la potencial escasa capacidad técnica de los reguladores de la red, que pueden asimilar cada capa a un mercado relevante dado, obviando otros factores.

\section{MARCO GENERAL DE LA REGULACIÓN INTERNACIONAL DE LA RED: GOBERNANZA DE INTERNET}

Cuando se habla de regulación internacional de Internet, no existe un único instrumento jurídico que regule a la red, ni todos ellos tienen la misma naturaleza. Por una parte, cada capa de la red partió siendo regu-

3. Más información sobre el caso en el siguiente enlace: <http://derechoaleer.org/ blog/20 I I/o8/efecto-leakymails-cientos-de-sitios-bloqueados.html>. 
lada, además de su código, mediante instrumentos específicos, atendido el origen histórico e institucional de cada una de ellas.

En la capa de infraestructura, la regulación internacional surgió a propósito de tratados internacionales respecto de las redes internacionales de telégrafos a fines del siglo XIX. En la capa de protocolos y aplicaciones, fueron surgiendo una serie de arreglos voluntarios alejados de las herramientas tradicionales del derecho internacional público. Por último, en la capa de contenidos se entrecruzan acuerdos internacionales de derechos humanos con la regulación de la propiedad intelectual y el comercio internacional. Este tema, que constituye el núcleo del presente trabajo, será abordado con más detalle en el subtítulo siguiente.

Este sistema regulatorio, donde conviven muy diversas instituciones, foros internacionales e instrumentos normativos de diversa naturaleza, configura un entramado institucional conocido como gobernanza de Internet, que trasciende los límites tradicionales del derecho internacional público, al configurar en su seno elementos que separan a la gobernanza de Internet de otros regímenes jurídicos internacionales. Entre esos elementos, destaca especialmente uno: los Estados, sujetos por excelencia del derecho internacional público, no poseen el protagonismo absoluto con el que cuentan en otros foros o normas internacionales.

En efecto, en los espacios de gobernanza de Internet conviven modelos bilaterales y multilaterales, donde los Estados aún son actores preponderantes, con modelos multisectoriales, en que diversas partes interesadas deliberan en un pie al menos formal de igualdad con miras a buscar acuerdos, con otros dirigidos por actores técnicos como la IETF (Internet Engineering Task Force, que será tratado más adelante en el presente trabajo) donde el debate no es tan importante como la aplicación práctica de las tecnologías involucradas, lo que se grafica en la famosa frase: «Rechazamos a reyes, presidentes y las votaciones. Creemos en los consensos brutos y el código funcionando».4

A lo anterior cabe sumar una dimensión política que excede lo institucional. Ampliando la definición que brindamos en la introducción de este trabajo, Carlos Cortés define gobernanza de Internet, para efectos prácticos, como «la gestión y el control de una actividad en la que par-

4. Traducción libre de la frase «We reject: kings, presidents, and voting. We believe in: rough consensus and running code» (Russell, 2006). 
ticipan múltiples actores — públicos y privados - con intereses contrapuestos», añadiendo que «involucra el diseño y la administración de las tecnologías necesarias para mantener el funcionamiento de Internet y la aplicación de políticas sustanciales alrededor de esas tecnologías» (Cortés, 20I4).

Sin embargo, el mismo autor busca profundizar otro aspecto en su trabajo, enfatizando el juego de equilibrios de poder y el carácter de fenómeno social de este sistema, señalando que no necesariamente la gobernanza real de Internet sucede al interior de las instituciones tradicionales que se asocian a ella.

Añade Cortés que la espina dorsal de la gobernanza de Internet está dada por su configuración tecnológica, que sirve para regular la conducta de los usuarios (teniendo presente que «el código es ley») y para determinar el poder de los actores que toman parte en la gobernanza de Internet.

El trabajo analizado recoge en gran medida lo planteado por Laura DeNardis en su libro The Global War for Internet Governance, que plantea la discusión de arreglos de arquitectura técnica de Internet como arreglos de poder, señalando que una visión ingenua de la regulación tecnológica podría sugerir que las autoridades establecen los objetivos para que luego los expertos técnicos y los coordinadores implementen esos objetivos en los sistemas. Ese enfoque, señala DeNardis, falla al considerar la influencia directa de fuerzas como el mercado y la interrelación entre sociedad y tecnología.

En ese sentido, para evitar un análisis ingenuo de los procesos de gobernanza de Internet, hay que tener en cuenta la distribución inicial de poder en Internet, en que algunos gobiernos e instituciones desde los inicios de la red tuvieron - y mantienen- un rol predominante en la misma. También hay que tener en cuenta que la mayoría de la infraestructura que sustenta Internet está en manos privadas, lo que significa un poder directo sobre su funcionamiento (DeNardis, 20I4: I 2).

Finalmente, hacen notar, la mayoría de los autores, que los procesos de gobernanza de Internet tienen enfoques diversos, que nacen de relatos determinados. En algunos espacios el foco es el desarrollo que se puede obtener a través de Internet, en otros la seguridad, el comercio y la observancia de los derechos humanos, entre varios otros, donde sin embargo las decisiones adoptadas en un espacio afectan todo el sistema, como 
sucede en el caso de la regulación de derechos de propiedad intelectual (Mueller, 20IO: I35).

\section{UN ANÁLISIS DE LA REGULACIÓN Y FOROS INTERNACIONALES VINCULADOS A INTERNET DESDE SUS CAPAS}

El análisis de la regulación internacional de Internet y la gobernanza de Internet a partir de sus capas es una idea que subyace a estos fenómenos, y en muchos casos las estructuras regulatorias y de gobernanza obedecen a la estructura de capas de la red. Lo interesante de esta aproximación es que de esta forma es posible conceptualizar la regulación de Internet como un proceso que toma lugar en múltiples niveles, y no en una dimensión monolítica y unitaria.

Desde esa mirada, y adoptando el modelo de capas de Benkler, revisaremos la regulación internacional en sus tres niveles, de acuerdo al siguiente esquema, listando algunos de sus temas frecuentes (adaptado de Kapur, 2005: 3):

Capa de infraestructura

- Interconexión de dispositivos

- Regulación de espectro electromagnético

- Acceso universal

Capa lógica

- Estándares

- Sistema de nombres de dominio

- Distribución de direcciones IP

Capa de contenidos

- Contenidos basura (spam)

- Datos personales y financieros

- Propiedad intelectual

Para efectos de este trabajo, no abordaremos cada uno de los temas indicados dentro de las capas, sino solamente los más relevantes desde 
un punto de vista normativo. Además, y como ya adelantamos, nos centraremos en las instituciones y normas más relevantes bajo un prisma de importancia y de actualidad de los debates que se llevan a cabo en su seno.

\section{REGULACIÓN DE LA CAPA DE INFRAESTRUCTURA}

Internet consiste en alrededor de cuarenta mil redes distintas, vinculadas entre sí, lo que crea un nivel de complejidad sin precedentes para la administración y gestión de la red (Renzenbrink, 20I4).

Atendido que el desarrollo comercial de Internet comenzó alrededor del año I995, de la mano de las operadoras de telecomunicaciones, la solución regulatoria en la mayoría de los países fue considerar Internet como un servicio de telecomunicaciones no regulado para permitir el crecimiento y desarrollo de la red. Además, las operadoras basaban su modelo de negocios en el tráfico de minutos de voz sobre el cual viajaban las señales de los módems, lo que simplificaba el problema.

\section{LA UNIÓN INTERNACIONAL DE TELECOMUNICACIONES}

A nivel internacional, existe una agencia de la ONU que se encarga de las tecnologías de la información y telecomunicaciones, que es la Unión Internacional de las Telecomunicaciones (UIT), fundada en el año I865 bajo el nombre de Unión Telegráfica Internacional. Está integrada por Estados, los que mantienen el control de la organización, además de empresas y centros de estudios que pueden sumarse al trabajo de la UIT, pero sin derecho a voto.

Hoy en día el foco de la UIT está puesto en tres áreas: estandarización, radiocomunicaciones y desarrollo. En el apartado de estandarización, existe una unidad denominada Sector de Normalización de las Telecomunicaciones (ITU-T), que se dedica a la generación de estándares para las telecomunicaciones. En el apartado de desarrollo, se preocupa de aumentar los niveles de acceso a las tecnologías, y en el de radiocomunicaciones, a regular y promover estándares respecto del uso de espectro electromagnético.

Como veremos, en la regulación de la capa lógica existe otro cuerpo de estándares denominado IETF, que se dedica a la regulación de los 
protocolos de Internet, a partir del protocolo TCP/IP. El criterio para diferenciar ambos es justamente la capa de Internet en la que trabajan. Mientras ITU-T trabaja con estándares para facilitar las telecomunicaciones desde una perspectiva de capa física, por ejemplo, proponiendo un tipo de conectores específicos, la IETF trabaja en la capa de protocolos y aplicaciones, por ejemplo, definiendo un protocolo de descarga de archivos de Internet. Sin embargo, existen muchos espacios en que el trabajo de estos cuerpos de estándares se superpone.

Dentro de la UIT, la regulación de los estándares está entregada a la ITU-T, que en colaboración con otros cuerpos que fijan estándares como el Instituto de Ingeniería Eléctrica y Electrónica (IEEE) y la International Standards Organizations (ISO), los van definiendo. El instrumento para ello son las Recomendaciones ITU-T, que definen cómo las redes de telecomunicaciones se interconectan e interoperan. Estas recomendaciones no son vinculantes hasta que se adoptan en el derecho interno de los países.

Además del trabajo de estandarización, la UIT está gobernada en base a acuerdos adoptados en las Conferencias Mundiales de Telecomunicaciones Internacionales (en inglés, WCIT) y las Reuniones de Plenipotenciarios. Mientras en la primera se discuten principalmente aspectos sustantivos sobre telecomunicaciones, en la segunda se eligen autoridades y discuten temas orgánicos y otros asuntos relacionados, sin perjuicio de poder abordar aspectos de política de telecomunicaciones.

Desde una perspectiva de capas, el trabajo de la UIT es doblemente sensible, por cuanto su rol es sumamente importante como ordenador internacional de las telecomunicaciones y la infraestructura física de Internet, y al mismo tiempo porque la posibilidad de emplear ese poder sobre las infraestructuras para perseguir objetivos en otras capas de Internet amenaza la integridad de las mismas, como ya hemos señalado.

\section{LAS REGULACIONES INTERNACIONALES DE TELECOMUNICACIONES}

En ese sentido, el año 20 I 2 se efectuó la última WCIT y el aspecto que despertó mayor polémica tuvo que ver con la revisión de las Regulaciones Internacionales de Telecomunicaciones (en inglés, ITR), que es el tratado vinculante que busca facilitar la interconexión e interoperabilidad de las tecnologías en el mundo. 
Lo que despertó la polémica fue precisamente la posibilidad que los ITR interfieran con otras capas de la red, como la capa de contenidos, debido a que existían propuestas que apuntaban a la regulación de los correos electrónicos no deseados (spam) por esta vía, o que se alteren reglas de pago por transmisión de contenidos, lo que afectaría la transparencia de la red y el principio punto a punto de la misma (Brown, 20I2).

La versión de las ITR aprobadas (International Telecommunications Regulations, 20I2 $)^{5}$ sólo contenía un par de disposiciones cuestionables desde el punto de vista del principio de capas, en los artículos 5 A y 5 B, que se refieren a la seguridad de las redes y las comunicaciones no solicitadas. Sin embargo, el lenguaje en esos artículos es sumamente vago y no representa un riesgo mayor para los contenidos en la red. Además, un número importante de países no firmó finalmente el acuerdo, entre los que se encuentra Chile.

\section{OTROS PROCESOS DE REGULACIÓN PRIVADA: NEGOCIACIONES DE INTERCONEXIÓN}

Finalmente, existe una dimensión normativa separada de la UIT, que carece de un marco regulatorio fijo y consiste en los acuerdos de interconexión que alcanzan los proveedores de acceso a Internet, que operan en distintos niveles de la red, pudiendo clasificarse en operadores Tier I (operadores de grandes líneas de enlace internacional), Tier 2 (operadores de enlaces nacionales o regionales) y Tier 3 (los proveedores de acceso a Internet). Estos acuerdos carecen de un marco regulatorio distinto al derecho internacional privado, y sus tarifas se negocian libremente. Algunos autores apuntan a que este sistema genera inequidad en las con-

5. El acuerdo está disponible en <http://www.itu.int/en/wcit-I 2/Documents/final-actswcit-I2.pdf>. Los artículos referidos señalan lo siguiente: Artículo 5 A: «Security and robustness of networks. Member States shall individually and collectively endeavour to ensure the security and robustness of international telecommunication networks in order to achieve effective use thereof and avoidance of technical harm thereto, as well as the harmonious development of international telecommunication services offered to the public.» Artículo 5 B: «Unsolicited bulk electronic communications. Member States should endeavour to take necessary measures to prevent the propagation of unsolicited bulk electronic communications and minimize its impact on international telecommunication services. Member States are encouraged to cooperate in that sense». 
diciones de acceso a Internet y genera un subsidio a la conectividad de países desarrollados (Kapur, 2005).

\section{REGULACIÓN EN LA CAPA DE PROTOCOLOS Y APLICACIONES}

Sobre la capa de infraestructura opera la capa de protocolos y aplicaciones, que como ya vimos en el caso de Internet funciona sobre la base del protocolo TCP/IP, que es el que da forma a la topología actual de la red. Dentro de esta capa, la regulación proviene de múltiples fuentes, la mayoría de ellas de carácter privado o no gubernamental.

Para efectos de este trabajo, separaremos el análisis normativo de la regulación en esta capa entre estándares, sistema de nombres de dominio y la asignación de direcciones IP.

\section{LA IETF}

Respecto a los estándares, como ya adelantamos, además de ITU-T (que ya abordamos en la sección anterior) existe un cuerpo de estándares denominado Fuerza de Tareas de Ingeniería de Internet (Internet Engineering Task Force, IETF, en inglés), que es un grupo de participación abierta a todos los interesados, en los que se fijan estándares para seguridad en Internet, empaquetamiento de datos, ruteo de datos, entre otros, donde destaca la administración de los protocolos TCP/IP.

A pesar de su carácter no vinculante, la IETF tiene un vasto poder regulatorio sobre Internet en lo que respecta a protocolos y estándares técnicos. Su trabajo gira en torno a diversas áreas de trabajo, que coordinan la discusión entre sus miembros. La principal herramienta de política en la que se traduce el trabajo de la IETF es en los RFC, que significa literalmente «request for comments» (petición de comentarios), en que algunos de ellos son informativos, experimentales y otros son sancionados como estándares (Request for Comments, 20I4).

\section{LA WORLD WIDE WEB CONSORTIUM}

Otra organización relevante para la fijación de estándares de Internet es la World Wide Web Consortium $\left(\mathrm{W}_{3} \mathrm{C}\right)$, que desarrolla estándares que funcionan sobre los protocolos base de Internet, directamente en la capa 
de aplicación, y que derivan de la creación por parte de Tim Berners Lee de la World Wide Web, que no es más que la red de contenidos estructurados a partir de los estándares HTML y HTTP.

\section{LAS ODII}

La IETF funciona al alero de la Sociedad de Internet (Internet Society, ISOC, en inglés), que a su vez forma parte de un grupo de organizaciones que se conoce como comunidad técnica de Internet u ODii, que significa Instituciones de Internet Orgánicamente Desarrolladas (Mueller, 20 Iо: 2 I7), que engloban al IETF, que funciona al alero de ISOC, que a su vez interactúan con ICANN y los Registros Regionales de Internet. Por razones de espacio y relevancia, la historia de estas organizaciones no será abordada en este trabajo.

\section{ICANN Y LOS NOMBRES DE DOMINIO}

Respecto a la administración de nombres de dominio, este proceso está regulado por la Corporación de Internet para la Asignación de Nombres y Números (ICANN, su sigla en inglés). Antes de continuar la explicación en este punto, brindaremos una brevísima explicación sobre la naturaleza de los nombres de dominio y números IP.

Para que opere el Protocolo de Internet (IP), cada nodo de la red debe contar con una dirección que la identifique, que se conoce como número IP. Sin embargo, atendida la complejidad de identificar cada computador con un bloque de 4 números que van de o a 255 (por ejemplo: I92.I68.0.I), fue diseñado un sistema que permita traducir nombres comunes a esos números, que son lo que conocemos como nombre de dominio. Por ejemplo, el dominio google.cl conduce a la dirección IP I73.I94.42.2 I6 (Peña, 20I3).

En ese marco, ICANN administra el sistema de creación y administración de estos nombres de dominio, fijando reglas básicas para la operación del sistema, delegando la administración de los dominios de alto nivel (.com, .net, .cl, etcétera), y cautelando que cumplan estándares de operación que permitan su funcionamiento estable y seguro.

ICANN funciona en base a una comunidad de múltiples interesados, donde gobiernos, sector privado, sociedad civil y comunidad técnica tie- 
nen voz y representación en su directorio, que adopta las decisiones finales a su respecto. Entre los temas que le ha tocado regular recientemente a ICANN están el manejo de las bases de datos de nombres de dominio, la creación de nuevos dominios de alto nivel y un desafío en marcha es gestionar el traspaso de las funciones que aún conserva el Gobierno de Estados Unidos en relación con la gestión del archivo raíz de los nombres de dominio (NTIA, 20I4).

\section{ASIGNACIÓN DE NÚMEROS Y REGISTROS REGIONALES DE INTERNET}

Para que el protocolo TCP/IP funcione, los nodos de la red requieren contar con direcciones o números IP que los identifiquen en la red. El encargado global de distribuir estos números es la Autoridad para la Asignación de Números de Internet (en inglés, IANA), que es un departamento de ICANN.

Esta autoridad distribuye bloques de números IP a los Registros Regionales de Internet (en inglés, RIRs), que a su vez lo distribuyen en las regiones a los usuarios de estos números, que principalmente son empresas tecnológicas y prestadores de servicio de Internet.

IANA no tiene facultades de regulación, sino más bien aplica las decisiones de ICANN y los estándares de la IETF. En particular, desde hace algunos años viene implementando el nuevo estándar de direcciones IP conocido como IP v6, que debido a su agotamiento deja atrás el sistema de números antiguo (de cuatro bloques de o a 255) e implementa una nueva nomenclatura, que permitirá suplir la permanente demanda por nuevas direcciones IP. Su implementación depende no sólo de IANA, sino también del resto de operadores de la red, y muy en particular de los prestadores de servicio de Internet.

\section{CAPA DE CONTENIDOS}

Dentro de la capa de contenidos se encuentra prácticamente todo aquello que el usuario promedio percibe como Internet. Cuando se habla de la regulación de Internet, muchos piensan en primer lugar en esta clase de regulación.

A diferencia de las capas anteriores, donde el análisis normativo necesariamente termina enfocado en instituciones de gobernanza de Internet, 
la regulación internacional de contenidos en Internet viene dada por una serie de interfaces con otros sistemas normativos, que apuntan a diversos fines, tales como acuerdos de propiedad intelectual, delitos informáticos, comercio y derechos humanos. Haremos a continuación una breve reseña de la regulación en cada ámbito y de sus puntos críticos.

\section{PROPIEDAD INTELECTUAL}

Una de las discusiones clave en la capa de contenidos, y probablemente aquella donde el debate es más álgido, dice relación con la protección de la propiedad intelectual en Internet. La razón central para ello es que tradicionalmente las reglas de propiedad intelectual fueron diseñadas para controlar la reproducción de obras del intelecto humano, y las tecnologías de la información permiten y se basan en la reproducción masiva de contenidos.

En ese sentido, en esta materia existen regulaciones internacionales tradicionales, como el Convenio de Berna (I 886), que señalan reglas básicas sobre protección de la propiedad intelectual, en que sus sucesivas reformas fueron ampliando el espectro de estos derechos, consagrando principios como la protección automática de las obras intelectuales, lo que supone un problema regulatorio cuando bajo esa norma la gran mayoría de los contenidos que circulan por Internet están efectivamente protegidos por derechos de autor (Lessig, 2004: I40).

Sumado a lo anterior, la negociación de nuevos acuerdos de propiedad intelectual a partir de la década de los noventa vino a complicar más las cosas, mediante tratados, como el Acuerdo sobre los Aspectos de los Derechos de Propiedad Intelectual Relacionados con el Comercio o el Tratado de la OMPI sobre Derecho de Autor, que incluían varias medidas tendientes a mejorar la aplicación de estos derechos en Internet, sin considerar las consecuencias que esto podría tener para otros derechos y sin dejar tampoco satisfechas a la industria del entretenimiento.

Desde la década del 2000, han existido aún más intentos para hacer más efectiva la protección de derechos de propiedad intelectual en Internet, con diverso éxito. Mientras en el ámbito multilateral no se han alcanzado consensos significativos, países como Estados Unidos han negociado exitosamente acuerdos de libre comercio con sus socios comerciales, conteniendo regulaciones que abarcan la propiedad intelectual en 
Internet, estableciendo reglas sobre observancia en Internet y responsabilidad de intermediarios en Internet, entre otras.

\section{RELACIÓN DE LA PROPIEDAD INTELECTUAL CON OTRAS CAPAS DE INTERNET}

Además de la regulación relativa a la protección de los derechos de autor en Internet, también la protección marcaria se ha abierto camino en la red, especialmente en la capa de protocolos y aplicaciones, mediante la implementación de mecanismos de resolución de conflictos derivados del registro de nombres de dominio.

Para estos efectos, ICANN dispuso, con fecha 26 de agosto de 1999 (ICANN, I999), una política uniforme de resolución disputas de nombres de dominio (en inglés, UDRP), que dispone que todos los registradores deben cumplirla, y donde la mayoría de los tipos de disputas de nombres de dominio basados en marcas registradas deben resolverse mediante acuerdo, acción judicial o arbitraje, antes que un registrador procesa a cancelar, suspender o transferir un nombre de dominio.

El sistema UDRP además ha inspirado mecanismos de resolución de conflictos en los registradores de dominios locales (.ar, .cl y el resto de los nombres de dominio de nivel nacional o ccTLD), que no están obligados a usar el modelo en las mismas condiciones que los registradores de dominios de primer nivel (.com, .org, etcétera).

El problema que presentan estos sistemas desde un punto de vista de interacción de capas es que en algunas de estas decisiones, especialmente a nivel de ccTLD nacionales, se emplean criterios de evaluación de contenidos de los sitios web, afectándose el principio de integridad de las capas, al intervenir en la capa técnica con base en información existente en la capa de contenidos.

Una última intersección se da entre ciertos protocolos técnicos diseñados por el ITU-T, IETF o el $\mathrm{W}_{3} \mathrm{C}$ y la propiedad intelectual, desde dos enfoques. Uno, cuando el protocolo en sí obedece a una tecnología sujeta a patentes u otra clase de protección análoga, lo que limita la adopción de estándares en la industria, debido a la necesidad de negociar regalías para su uso. En ese sentido, tecnologías que definen a Internet, como el protocolo TCP/IP, HTTP y HTML, han sido compartidos libres de cualquier clase de propiedad intelectual sobre ellos. 
El otro enfoque es cuando se adoptan estándares que permiten un mayor control de Internet por parte de algunos actores, en aras de la propiedad intelectual. En ese sentido, la adopción reciente en la $\mathrm{W}_{3} \mathrm{C}$ del estándar EME (extensiones de medios encriptadas), ha sido ampliamente debatida por la sociedad civil, por entregarle un control excesivo de la web a los titulares de propiedad intelectual. ${ }^{6}$

Sumado a lo anterior, toda la discusión respecto a la regulación de la propiedad intelectual en Internet no sólo debe considerar estas normas, sino también aquellas relativas a derechos humanos, como veremos.

\section{DELITOS INFORMÁTICOS}

Los delitos informáticos tienen relación con múltiples capas de la red, aunque suele tratársele principalmente en la capa de contenidos. A nivel internacional, existe un instrumento internacionalmente reconocido para la tipificación y persecución penal de estos delitos, que es el Convenio sobre la Ciberdelincuencia, donde se propone un catálogo base de delitos informáticos y medidas de colaboración internacional en la materia.

Sin embargo, de los nueve delitos considerados en el Convenio, sólo los dos últimos, relacionados con la pornografía infantil y la infracción a la propiedad intelectual, pertenecen inequívocamente a la capa de contenidos. El resto, que comprende tipos de acceso ilícito, interceptación ilícita, ataques a la integridad de datos o del sistema, abuso de dispositivos y falsificación informática, puede involucrar además alguna de las otras dos capas, especialmente la de protocolos y aplicaciones, dependiendo de las herramientas empleadas o el resultado obtenido con el delito. Existe un protocolo adicional a este convenio, que trata sobre delitos racistas y de odio, que está vinculado con la capa de contenidos.

Las discusiones sobre seguridad en Internet, donde el combate al cibercrimen forma parte central, cruzan de manera transversal las diferentes capas de la red. Lo que cabe en estos casos es radicar la aplicación de cada regulación específica en la capa que corresponde, para garantizar

6. La Electronic Frontier Foundation publicó un artículo al respecto, disponible en <https://www.eff.org/deeplinks/20I3/ıo/lowering-your-standards>. 
el principio de separación de capas y la eficiencia e integridad de la red, como veremos más adelante en este trabajo.

\section{COMERCIO INTERNACIONAL}

El rol de la regulación del comercio internacional y, más específicamente, de los acuerdos de libre comercio en la regulación de Internet, se está volviendo cada vez más significativo. Además de la propiedad intelectual, a la que ya nos referimos, diversos capítulos y normas sobre protección de inversiones, telecomunicaciones y protección de datos personales afectan las discusiones internacionales sobre gobernanza de Internet en múltiples capas.

Hoy en día se discuten temas relativos a la circulación de la información en el marco de la liberalización del tránsito de bienes y servicios. Sin embargo, esas disposiciones persiguen no sólo facilitar la circulación de la información o la libertad de expresión, sino también desregular el tráfico transfronterizo de datos personales, afectando de esta forma la protección de la vida privada de las personas, e invirtiendo en la práctica la regla general instalada por principios internacionales de derechos humanos al proponer un paradigma de libre circulación de datos por sobre la protección a la privacidad de las personas (Kelsey y Kilic, 20I4: 2I).

\section{DERECHOS HUMANOS}

La regulación internacional de derechos humanos es también un punto crítico a considerar en la capa de contenidos, toda vez que los principios contenidos en sus instrumentos internacionales tienen un nivel de desarrollo considerable, al punto de contar con sistemas de observancia efectivos para su protección.

A nivel global, los instrumentos jurídicos más significativos son la Declaración Universal de Derechos Humanos (I948), y el Pacto Internacional de Derechos Civiles y Políticos (1976), que contienen protecciones específicas a la libertad de expresión, privacidad, no discriminación, acceso al conocimiento y otros derechos relevantes en Internet. Algunos sistemas regionales de protección, como el interamericano y el europeo, contienen mayor desarrollo de estos derechos, y sanciones ante la infracción de los mismos. 
En la práctica, muchas de las discusiones en materia de propiedad intelectual, libre comercio y delitos informáticos se suscitan a propósito de la falta de balance entre éstos y el régimen internacional de derechos humanos en vigor. Las normas de propiedad intelectual necesitan mayor equilibrio con el derecho de acceder al conocimiento y de libertad de expresión. Las normas de delitos informáticos generan también tensiones con los derechos a la no discriminación, la libertad de expresión y la privacidad, producto de las medidas abusivas para investigar estos delitos; $y$ las disposiciones contenidas en tratados de libre comercio muchas veces ponen en entredicho el derecho a la privacidad y libertad de expresión.

Desde un punto de vista de separación de capas, diversos derechos humanos operan en cada una de ellas. Además de su aplicación en la capa de contenidos, los derechos a la no discriminación y acceso al conocimiento están íntimamente vinculados con la capa física, que es donde las discusiones de conectividad están radicadas. En la capa de protocolos y aplicaciones, muchas veces puede verse afectada la libertad de expresión, producto de regulaciones que fallan al entender los aspectos técnicos de Internet.

Sumado a lo anterior, en el seno de Naciones Unidas también se ha presentado la discusión sobre la aplicación de derechos humanos en Internet, que resultó en una declaración señalando que «los derechos de las personas también deben estar protegidos en Internet, en particular la libertad de expresión, que es aplicable sin consideración de fronteras y por cualquier procedimiento que se elija» (Asamblea General de la ONU, 20I2), despejando cualquier duda a este respecto.

\section{CONSIDERACIONES FINALES}

Tras realizar un análisis general de diversos organismos y regulaciones internacionales de Internet, queda preguntarse qué clase de relaciones, soluciones normativas y aplicaciones concretas pueden desprenderse de la teoría de separación de las capas y las consideraciones realizadas.

Respecto a cómo las capas determinan relaciones de poder e institucionales, podemos ver claramente que el ámbito específico de acción de cada uno de los órganos y regulaciones internacionales vinculados a Internet tiende a estar delimitado por la capa en la que actúa, bajo el modelo simplificado de capas de infraestructura, lógica y de contenidos. 
En efecto, cada capa posee una configuración característica: mientras en la de infraestructura los actores más importantes son los Estados (a través de la ITU) y el sector privado (mediante arreglos de tráfico), en la lógica son los sectores técnicos quienes conducen los procesos de gobernanza (mediante ICANN y otros organismos), mientras que en la de contenidos existe un marco mucho más diversos donde se combinan regulaciones sobre propiedad intelectual, delitos informáticos, comercio internacional y derechos humanos, con múltiples tensiones derivadas del balance de este último tema con los primeros tres.

Esta configuración de instituciones y regulaciones se ha ido generando a la imagen de las capas de Internet, y resulta conveniente mantenerlas funcionando de esta forma, con competencias definidas en base a éstas, procurando evitar el cruce de competencias y el tratamiento de la red como una tecnología monolítica al servicio de intereses específicos.

En relación con las soluciones normativas y aplicaciones concretas, mientras en el plano nacional los sistemas jurídicos regulan con bastante detalle diversos fenómenos relacionados con Internet, lo que permite un análisis acucioso y detallado de normas a la luz del principio de separación de capas, en el plano internacional encontramos un escenario mucho más complejo, donde la vaguedad de ciertos términos y la falta de regulación efectiva deja un espacio acotado para proponer soluciones normativas, acotándolas a principios o reglas generales en materia de regulación de Internet.

En ese sentido, existen dos principios generales que asoman como una directa aplicación de la teoría de separación las capas al campo de la regulación internacional de Internet: el principio de limitación de responsabilidad de intermediarios y el de neutralidad de la red.

\section{PRINCIPIO DE LIMITACIÓN DE RESPONSABILIDAD DE INTERMEDIARIOS}

El primer principio es una aplicación directa del principio de separación de capas, y consiste en dotar a ciertos intermediarios de Internet de una defensa legal frente a las actividades que se lleven a cabo en sus servicios o redes. Esta defensa puede estar acotada a un tipo de actos (infracciones a propiedad intelectual) o ser una herramienta genérica, aplicable a todo tipo de casos.

Si este principio ya es importante respecto a proveedores de servicios 
de contenido, como una red social o un sitio que facilite el intercambio de contenidos generados por usuarios, lo es mucho más cuando se aplica a intermediarios que se encuentran en otra capa, que puede ser técnica, de infraestructura o - como suele acontecer- una mezcla de ambas, respecto de los servicios que provee un prestador de servicios de conexión a Internet. Esta importancia viene dada por la forma en que la limitación de responsabilidad de intermediarios puede reforzar a su vez el principio de integridad de las capas.

En el ámbito internacional, este principio se ha implementado a propósito de reglas internacionales de propiedad intelectual, especialmente en acuerdos de libre comercio que ha negociado y suscrito Estados Unidos con varios países, entre los que se encuentra Chile, y que obliga a los países que los suscriben a establecer un mecanismo de limitación de responsabilidad hasta que el intermediario recibe una notificación sobre la actividad ilícita (Lara y Vera, 20 2: 3).

\section{PRINCIPIO DE NEUTRALIDAD DE LA RED}

El segundo principio es el de neutralidad de la red, que impide que los prestadores de servicio de Internet puedan discriminar entre el tráfico de datos de sus usuarios para privilegiar unos contenidos sobre otros.

A favor de la neutralidad de la red, se argumenta que el protocolo TCP/IP funciona en base a los principios de punto a punto y mejor esfuerzo, lo que redunda en una red transparente y neutral al contenido, lo que es plenamente concordante con el principio de integridad de las capas, que impide manipular una capa (en este caso, la lógica) para obtener resultados en la de contenidos, alterando el funcionamiento de la red mediante el cruce entre capas.

\section{NETMUNDIAL}

El principio de la neutralidad en la red aún carece de consagración internacional, y la primera ley nacional sobre la materia data del año 2010 (Lara, Vera y Viollier, 20I4: 3-I3), por lo que aún resta un tiempo considerable para que se consolide y reconozca a nivel global.

Sin embargo, el principio de la neutralidad ya está siendo discutido en foros internacionales, entre los que destaca la reunión NetMundial, 
celebrada en Sao Paulo en abril del año 20I4, de carácter multisectorial, y que desembocó en una declaración de consenso en la que existen menciones específicas a la protección de los intermediarios y la neutralidad de la red. ${ }^{7}$

Sobre el primer punto, la declaración NetMundial señala que las limitaciones a la responsabilidad de intermediarios deben ser implementadas en una forma que respeten y promuevan el crecimiento económico, la innovación, la creatividad y el libre flujo de información. Cierra este punto la declaración llamando a cooperar entre todas las partes interesadas para prevenir actividades ilegales, consistentes con el derecho al debido proceso.

Si bien el lenguaje es bastante vago, es importante que una declaración de este tipo haya hecho mención expresa a este punto. La declaración NetMundial también hace una mención a la neutralidad de la red, pero en este caso solamente se consigna que es una discusión que deberá ser abordada en el futuro, donde existen visiones divergentes, y se recomienda seguirla tratando en foros tales como el próximo Foro de Gobernanza de Internet.

\section{EL FORO DE GOBERNANZA DE INTERNET COMO UN FORO TRANSVERSAL A LAS CAPAS}

Además de NetMundial, existen otros espacios donde estos temas son regularmente discutidos. Sin lugar a dudas, el más importante hoy en día es el Foro de Gobernanza de Internet (IGF, por su nombre en inglés), que está vinculado a la ONU y nació como producto de la Agenda de Túnez para la sociedad de la información, que a su vez fue fruto de la Cumbre Mundial sobre la Sociedad de la Información, consistente en un par de conferencias gestadas por las Naciones Unidas en los años 2003 y 2005 .

El IGF nació de la Agenda de Túnez, antes citada, que en su párrafo 72 pidió un nuevo foro de diálogo multisectorial, que desde entonces se ha reunido anualmente para discutir varios aspectos de políticas públicas relativos a Internet, de manera no vinculante ni resolutiva.

La particularidad de este foro es que intenta integrar todas las discu-

7. Declaración disponible en el siguiente enlace: <http://netmundial.br/wp-content/ uploads/2OI4/o4/NETmundial-Multistakeholder-Document.pdf $>$. 
siones, de distintos órganos involucrados en la gobernanza de Internet, convirtiéndose en un foro que cruza transversalmente las capas de la red, en donde se han iniciado y continuado debates sobre prácticamente todos los temas tratados en este trabajo.

Si bien el IGF, al intentar reunir todas las discusiones, puede parecer un riesgo para el principio de la integridad de las capas, en realidad representa una oportunidad para empujar y promover este principio, ya que debido a su naturaleza no resolutiva permite concentrar algunas discusiones mientras la toma de decisiones se mantiene descentralizada en cada capa.

\section{CONCLUSIONES}

Internet es una tecnología compleja, que para efectos de su análisis puede ser dividida en múltiples capas que den cuenta de los procesos técnicos que permiten su existencia, combinando infraestructura física, protocolos técnicos y los contenidos que circulan por ella.

El análisis por capas es perfectamente aplicable a la regulación de la Internet, revisando en cada capa las regulaciones e instituciones relevantes para su existencia y funcionamiento.

A partir de proceso de análisis, se puede también concluir que el respeto de la integridad de estas capas es un principio deseable para la regulación de Internet, considerando el código (la estructura técnica) al momento de crear normas que rijan la red, con el objetivo de preservar su funcionamiento.

En el contexto internacional, este análisis por capas es también posible, tomando en consideración que la regulación suele provenir de diversos órganos y procesos que poseen distintas calidades jurídicas, por lo que su división en capas permite agrupar estos órganos y procesos de acuerdo a la función que cumplen en la red.

A diferencia de las capas de infraestructura y lógica, donde la presencia de normas jurídicas tradicionales es relativamente escasa y existe un número de órganos técnicos que se hacen cargo de regular estas capas mediante código, en la capa de contenidos intervienen numerosas regulaciones que no fueron específicamente concebidas para Internet; queda el desafío de armonizarlas, con especial consideración a los derechos humanos en juego. 
Muchos temas quedan fuera de este trabajo. Entre ellos, un análisis de la regulación tecnológica y el código desde una perspectiva filosófica o de teoría del derecho, una explicación más exhaustiva de los órganos, procesos e instrumentos jurídicos vinculados a la gobernanza de Internet, y un análisis exhaustivo de las interacciones de temas que cruzan diversas capas de la red, desde una perspectiva de integridad y minimización de cruce de éstas.

Confiamos, sin embargo, en que las referencias y sistematización ofrecidas sirvan de ayuda a quien quiera introducirse en el análisis de la regulación internacional de Internet, para avanzar en el estudio y comprensión de la materia.

\section{REFERENCIAS}

Bailey, Jane (2004). "Of Mediums and Metaphors: How a Layered Methodology Might Contribute to Constitutional Analysis of Internet Content Regulation", Manitoba Law Journal, I97: I4-I7.

Barlow, John Perry (I996). «Declaración de independencia del ciberespacio». Disponible en <https://projects.eff.org/ barlow/DeclarationFinal.html>.

Benkler, Yochai (2000). "From Consumers to Users: Shifting the Deeper Structures of Regulation Toward Sustainable Commons and User Access». Federal Communications Law Journal, 52: 552-579.

Brown, Deborah (20I2). "What to Watch at WCIT». Disponible en $<$ https://s3.amazonaws.com/access.3cdn.net/di 6ff8602d9ra6erad_ ezm6b9ov2.pdf $>$.

Cortés, Carlos (2014) «La gobernanza de Internet: La trampa de las formas». Disponible en <http://www.palermo.edu/Archivos_content/20I4/derecho/agosto/internet/cele_gobernanzadeinternet.pdf $>$.

DeNardis, Laura (20I4). The Global War for Internet Governance. New Haven: Yale University Press.

ICANN (1999). Uniform Domain Name Dispute Resolution Policy. Disponible en <https://archive.icann.org/en/udrp/udrp-policy-240ct99. htm>.

ITU (20I2). International Telecommunications Regulations.20I2. Disponible en <http://www.itu.int/en/wcit-I 2/documents/final-actswcit-I 2.pdf>. 
Kapur, Akesh (2005). Internet Governance: A Primer. Nueva Delhi: Reed Elsevier India.

Kelsey, Jane y Burcu Kilic (20I4). "Briefing on US TISA Proposal on E-Commerce, Technology Transfer, Cross-Border Data Flows and Net Neutrality». Disponible en <https:/data.awp.is/data/filtrala/I $5 /$ analisis.cleaned.pdf $>$.

Lara, Juan Carlos y Francisco Vera (20I2). «Responsabilidad de Los Prestadores de Servicios de Internet». Disponible en <https://www. derechosdigitales.org/wp-content/uploads/ppo3.pdf $>$.

Lara, Juan Carlos, Francisco Vera y Pablo Viollier (20I4). «Estado de Internet en Chile: Aspectos generales, regulación y actores relevantes». Disponible en <https:/www.derechosdigitales.org/wp-content/ uploads/PPo6.pdf $>$.

Lessig, Lawrence (200I). The Future of Ideas: The Fate of the Commons in a Connected World. Nueva York: Random House.

-. (2004). Free Culture: How Big Media Uses Technology and the Law to Lock down Culture and Control Creativity. Nueva York: Penguin Press.

-. (2006). Code: Version 2.0. Nueva York: Basic Books.

Mueller, Milton (2010). Networks and States: The Global Politics of Internet Governance. Information Revolution and Global Politics. Cambridge: MIT Press.

NTIA (20I4). «NTIA Announces Intent to Transition Key Internet Domain Name Functions». Disponible en <http://www.ntia.doc.gov/ press-release/20 I 4/ntia-announces-intent-transition-key-internet-domain-name-functions $>$.

O'BRIEN, Danny (2OI4) «Lowering your Standards: DRM and the Future of the $\mathrm{W}_{3} \mathrm{C}$ ». Disponible en <https://www.eff.org/deeplinks/20 I3/Io/ lowering-your-standards $>$.

OECD (20II). «OECD Council Recommendation on Principles for Internet Policy Making». Disponible en <http://www.oecd.org/internet/ ieconomy/49258588.pdf>.

PeÑa, Paz (2013). Cómo funciona Internet. Santiago: ONG Derechos Digitales.

REED, David (2006). «Critiquing the Layered Regulatory Model». Journal on Telecommunications and High Technology Law, 4: 296-298. Renzenbrink, Tessel (20I4). «Internet @ The Physical Layer». Dis- 
ponible en <http://www.techthefuture.com/technology/internet-thephysical-layer/>.

Solum, Lawrence y Minn Chung (2004). "The Layers Principle: Internet Achitecture and the Law». Notre Dame Law Review, 79 (3): 8I 5-948.

Werbach, Kevin D. (2002). "A Layered Model for Internet Policy». Journal on Telecommunications and High Technology Law, I (37): I6-26.

Wikipedia (20I4). «OSI Model». Disponible en <http://en.wikipedia. org $/$ w/index.php?title=OSI_model\&oldid $=636349030>$.

-. (20I4). «Request for Comments». Disponible en <http://es.wikipedia. org/w/index.php?title=Request_for_Comments\&oldid=75 $525670>$.

WhitT, Richard S. (2004) «A Horizontal Leap Forward: Formulating a New Communications Public Policy Framework Based on the Network Layers Model». Federal Communications Law Journal, 56 (3): 6I4-632.

Yoo, Christopher (2013). «Libertad de expresión y el mito de Internet como una experiencia no intermediada». Revista Chilena de Derecho y Tecnología, 2 (I): I I-I I I.

\section{SOBRE EL AUTOR}

Francisco Vera Hott es abogado. Licenciado en Ciencias Jurídicas y Sociales por la Universidad de Chile. En su carrera ha asesorado a diversas organizaciones de la sociedad civil y de gobierno en relación con la regulación nacional e internacional de Internet. Su correo electrónico es $<$ fvera@prys.cl>y su dirección postal es Nueva York 9, piso I4, Santiago, Chile.

Este trabajo fue recibido el 3 de diciembre y aprobado el 3 I de diciembre de 2014 . 
